\title{
Awareness in P2P groupware systems: a convergence of contextual computing, social media and semantic web
}

\author{
Alex Poulovassilis (ap@dcs.bbk.ac.uk) and Fatos Xhafa (fatos.@lsi.upc.edu), \\ London Knowledge Lab, 23-29 Emerald St., London, UK
}

\section{Introduction}

Collaborative work through virtual teams so as to undertake a common group project is a significant mode of collaboration in online learning. This kind of collaboration requires efficient communication and sharing of information between group members. P2P technologies can potentially foster more support for collaboration than centralized approaches as group members can interact directly with their peers in order to provide additional scaffolding or social support.

Awareness is a vital aspect of collaborative systems, and refers to knowledge provided by the system to group members about what other group members are doing at present same time and what they did in the past [4]. Since collaboration usually takes place throughout the lifespan of a project, the temporal dimension is also very important. For example, the time needed for completion of group tasks can be decreased by enabling faster information and knowledge transfer between group members.

Awareness systems for online collaborative work have been proposed since in early stages of Web technology $[23,1,21]$. More recently, research has focussed on using new paradigms, such as P2P systems, to achieve fully decentralized, ubiquitous groupware systems and awareness in such systems $[2,16,15,6,8,7,5,9$, 11, 19]. The use of Service-Oriented Architecture, and specifically OSGi technology, has also been recently explored for supporting P2P group collaboration of mobile peers [3]. However, to date, service-oriented approaches have not supported a full-featured awareness model.

Several challenges arise in developing groupware and awareness mechanisms in fully decentralized P2P systems:

- it is more difficult to achieve a consistent view of the groupware global state from the local states of the group members;

- P2P systems are primarily aimed at supporting explicit peer-to-peer communication, while group collaboration requires many-to-many communication;

- issues inherent to P2P systems such as their dynamicity and heterogeneity need to be dealt with. The awareness functionality needs to provide appropriate mechanisms, such as propagation and replication of information, to ensure provision of awareness as peers leave and join the system. Data representation within the $\mathrm{P} 2 \mathrm{P}$ middleware needs to allow encoding of the application-specific information required to support the awareness functionality e.g. information about group members, resources, activities and processes, the project workflow, etc. Lightweight mechanisms are essential for mobile peers with limited computational resources. Coarse-grained information presentation (overviews and summaries) is also necessary to allow adaptation to a peer's computational resources for resource constrained devices.

- P2P systems are pervasive and ubiquitous in nature, thus requiring contextualized awareness. In many situations, the chronological representation of a temporal stream of events is not sufficient and event transformation may also be necessary. This may involve extension of the event information with other related contextual information, or composition of events to yield new more complex events. Awareness 
information should be available at different levels of granularity, ranging from overviews to fully detailed reports. For instance, for a latecomer to the group it is more useful to receive overviews and summaries on group activities to date rather than full reports.

Awareness mechanisms in $\mathrm{P} 2 \mathrm{P}$ systems have generally been implemented as part of applications, thus having the limitation of being application dependent. There has been little research in endowing P2P collaborative systems with generic, application- and platform-independent awareness mechanisms. Moreover, the focus has been on providing information about individuals' activities (e.g. in the form of simple notifications) rather than on supporting group processes so as to accomplish a common group project.

To this end we are exploring the provision of service-based group awareness capabilities within the P2P middleware on top of which groupware applications can be developed [22]. Also, unlike existing approaches that provide awareness information directly from streams of events, we are proposing event transformation capabilities to achieve presentation of information in different formats, tailored to group members' needs and to their computational resources.

The provision of service-based group awareness capabilities in $\mathrm{P} 2 \mathrm{P}$ systems is a setting that represents a convergence of pervasive computing (since network peers may be mobile as well as fixed), social media (since groupware is founded on synchronous and asynchronous communication between peers), and the semantic web (using languages such as $\mathrm{RDF} / \mathrm{S}$ to represent and reason with the diverse range of information required by the P2P middleware and the awareness services).

We next discuss some major types of group awareness. Our computational model for supporting group awareness is described in Section 3. In Section 4 we discuss how the proposed model can support an important type of online learning, namely distributed scenario-based learning. We conclude in Section 5.

\section{Types of Group Awareness}

We summarize below some major types of group awareness (see also $[4,23]$ in the context of web-based groupware), which are supported by the proposed computational model.

Activity awareness aims to provide information about progress on the accomplishment of project tasks by individuals and the group as a whole. It comprises knowing about actions taken by members of the group according to the project schedule, and synchronization of activities with the project schedule.

In project-based work, a project typically requires the enactment of a workflow. The workflow comprises a set of tasks and precedence relationships relating to their order of completion. Process awareness tracks the state of the project workflow, providing group members with a partial view (what they are each doing individually) and a complete view (what they are doing as a group).

Communication awareness relates to message exchange, and synchronous and asynchronous discussion forums. It supports awareness of P2P communication (a peer can establish a direct communication with another peer), awareness of the creation and lifetime of discussion forums (so that other peers can be aware of, and eventually join, these), and awareness of new messages posted in the discussion forum, replies, etc.

Context awareness refers to information about the time, location and environment in which group members perform actions, and to the profiles of group members and of the group as a whole. In a networked environment, other features are also relevant to context awareness such as network connectivity, communication costs and bandwidth, and the social situation of members. The latter includes emotional awareness, aiming to provide computational support in recognizing and representing members' emotional states.

Availability awareness provides group members with information about members' and resources' availability. The former is necessary for establishing synchronous collaboration while the later is useful for supporting members' tasks requiring specific resources. Availability awareness encompasses knowing who is available at what time (via members' profiles, which include personal calendars), and what resources are available at what time (via scheduling information about resources' usage). 


\section{Our Event-based Awareness Model in P2P Groupware Systems}

We propose an RDF-based superpeer network model $[10,20,13]$ for supporting group awareness in P2P groupware systems. In this model, the network consists of several peergroups. The peers of each peergroup are connected to a single superpeer. There is frequent local communication between peers within a peergroup, and less frequent global communication between superpeers. Superpeers serve as coordinators of the overall network while other peers represent group members at the network "edge" - superpeers will typically be fixed networked computers while peers may be fixed or mobile more resource-constrained devices.

Peergroups form in order to undertake group projects - thus peers may join or leave a peergroup at any time. Information about the groups processes is distributed and replicated at all peers of the peergroup. This enables efficiency due to local access to data and support of failures e.g. when peers leave or are temporarily disconnected. Peers' operations and control information is forwarded to their superpeer which manages the replication and consistency of information within the peergroup. Our model aims to support both stand-alone mobile peers and mobile peers that are attached to other fixed peers through lightweight mechanisms and summary services (see [22] for details).

All peers (and supeerpeers) have query, storage and update capabilities over their local data, which is represented using RDF/S. Each peer and superpeer hosts a fragment of an overall global RDFS schema, and its local RDF data conforms to this fragment. Each superpeer's RDFS schema is a superset of its peergroup's individual RDFS schemas. Each superpeer defines access privileges over the classes and properties in its RDFS schema. More fine-grained access privileges are also possible on specific RDF resources and properties. These facilities allow a superpeer to specify which data can be shared with other superpeers, outside the peergroup (within the peergroup all data is shared and replicated).

Each superpeer has Event-Condition-Action (ECA) rule processing capabilities. ECA rules hosted at the superpeer can be used to:

1. achieve replication and consistency of distributed group data and processes;

2. automate the generation and propagation of global overviews and summaries from detailed information and local summaries received from individual peers;

3. automate the receipt of awareness information by peers according to their current status, status of the project they are participating in, their preferences, and their context; this may be activity, process, communication, context, and availability awareness information, as discussed earlier; and it can be received in both "passive" and "active" mode (with the former awareness information is delivered to group members without requiring any specific actions on their part, while with the latter it requires group members to undertake specific actions to request awareness information).

Updates initiated at a peer site, affecting the peer's local data, are first executed locally and are then notified to the superpeer. The superpeer then decides whether the update operation triggers an ECA rule (or rules) causing the stated rule actions to be propagated to other peers in the peergroup (this is the P2P ECA rule execution model proposed in [13] and explored further in [14]).

\section{Awareness Support to Distributed Scenario-based Learning}

We consider here how group awareness can support an important type of learning, namely the scenariobased learning (SBL) $[17,18]$. In this learning setting, the students are given a real learning context that requires acquiring and applying knowledge and practice skills in order to forge a path through to completion of a learning activity. SBL arises quite naturally in our online learning model of Project-Based Learning (PBL) [24] in which learning is seen as a process-oriented activity through which learners build their knowledge by solving problems, accomplishing tasks and projects. SBL can be thus seen as a particular case of PBL. In a distributed P2P environment, group awareness is very important during the collaboration process in which a group of students undertake the tasks within their learning scenario. 
In our context of PBL, a learning scenario is defined as a set of tasks, forming a subgraph within the project workflow. In a distributed $\mathrm{P} 2 \mathrm{P}$ environment, the set of tasks should: (a) be formally defined as a learning activity; for example, in the domain of software engineering, as a set of tasks contributing towards a group software development project; (b) be assigned to members of the group; (c) be coordinated in order to maintain the project timeline and inter-task dependencies; (d) have the peer resources that are needed for task accomplishment be made available by individual group members and managed by the system; (e) be tracked and monitored by the system so as to have knowledge of the status of the tasks; and (f) have the status of each task updated as appropriate, moving through different states such as "pending assignment", "assigned", "in progress", "delayed", "awaiting for another task completion", "completed" etc.

Our proposed awareness model can effectively support aspects (a)-(f) above. Aspects (a), (b) and (f) are supported by activity awareness. The group superpeer has knowledge of the group members, project tasks, project workflow, and the responsibilities assigned to each group member. Aspects (c) and (e) are supported by process awareness and communication awareness. Peers notify the superpeer regarding their status and their progress on individual tasks. Such information can be propagated (via appropriate ECA rules) by the superpeer to all peers. Superpeers can also generate as necessary overviews and summaries from the information being sent to them by peers, and can propagate this to individuals (again via appropriate ECA rules). Aspect (d) is supported by availability awareness: peers notify the superpeer regarding their resource requirements and the availability of their resources. The superpeer dynamically manages resource allocation and scheduling, and this information is dynamically propagated to all peers.

SBL can be seen also as context-based learning by placing the set of tasks of the scenario within their context (time, workspace, member, ...) and can thus be further supported by context awareness. Finally, many studies in CSCL have shown that learning scenarios are effective learning methods for motivating learners and this can be supported by emotional awareness within our model.

The Open University of Catalonia [12] is a concrete example where distributed scenario-based learning is used to achieve learning goals. Many subjects are designed as PBL and use SBL for achieving particular learning goals. For instance, a group of students assigned a software development project use SBL for developing and integrating the different components of their software application.

\section{Conclusions and Future Work}

In this position paper we have discussed the support of awareness in $\mathrm{P} 2 \mathrm{P}$ groupware systems. We have focussed particularly on support of groups undertaking common projects in a learning setting, although our approach is also relevant more broadly to teamwork settings in industry, health-care etc. We have argued that provision of service-based group awareness capabilities in $\mathrm{P} 2 \mathrm{P}$ systems is a setting that represents a convergence of pervasive computing, social media, and the semantic web. We have discussed how the proposed awareness model can support an important type of online learning, namely distributed scenario-based learning. Future work includes design of the awareness primitive operations and services, and evaluation of how our model can be integrated into existing groupware systems and distributed learning scenarios.

\section{References}

[1] R. Bentley, T. Horstmann, K. Sikkel and J. Trevor. Supporting Collaborative Information Sharing with the WWW. The BSCW Shared Workspace System, The World Wide Web Journal, 63-73, 1995.

[2] P. Busetta and M. Merzi. Approach to the Integration of Peer-to-Peer Systems with Active Environments, WOA 2003: 42-48.

[3] D.G. Galatopoullos, D.N. Kalofonos, and E.S. Manolakos. A P2P SOA enabling group collaboration through service composition. In Proc. of the 5th Int'l Conference on Pervasive Services, 111-120, 2008.

[4] C. Gutwin, S. Greenberg, and M. Roseman. Workspace Awareness in Real-Time Distributed Groupware: Framework, Widgets, and Evaluation. BCS HCI 1996: 281-298. 
[5] T. Kawashima, J. Ma. TOMSCOPA synchronous P2P collaboration platform over JXTA. Proc. of the Int'l Workshop on Multimedia Network Systems and Applications, 85-90, 2004.

[6] R. Kurmanowytsch, E. Kirda, C. Kerer, S. Dustdar. OMNIX: A topology-independent P2P middleware, LNCS, vol. $75,47-56,2003$.

[7] Y. Li, J. Bu, C. Chen, X. Xu. Reliable Communication Based on P2P Architecture on Real-Time Collaborative Editing System. The 8th Int'l Conference on CSCW in Design, 244 - 249, 2004

[8] J. Ma, M. Shizuka, J.Lee, R Huang. A P2P Groupware System with Decentralized Topology for Supporting Synchronous Collaborations. Proc. of the Int'l Conference on Cyberworlds, 54-61, 2003.

[9] M. Margaritis, C. Fidas, N. Avouris, V. Komis. A Peer-to-Peer Architecture for Synchronous Collaboration over Low-Bandwidth Networks. University of Patras, TechRep 26500, Greece.

[10] W. Nejdl, B. Wolf, C. Qu, S. Decker, M. Sintek, A. Naeve, M. Nilsson, M. Palmr, and T. Risch. Edutella: A P2P Networking Infrastructure Based on RDF. Proc. of the 11th World Wide Web Conference, 604-615, 2002.

[11] Y. Oasis, S. Abdala, A. Matrawy. A Multilayer P2P Framework for Distributed Synchronous Collaboration. IEEE Internet Computing, 33-41, 2006

[12] Open University of Catalonia: http://www.uoc.edu

[13] G. Papamarkos, A. Poulovassilis, and P.T. Wood. Event-Condition-Action Rules on RDF Metadata in P2P Environments. Computer Networks, 50(10):1513-1532, 2006.

[14] G. Papamarkos, A. Poulovassilis, and P.T. Wood. Performance Modelling of Event-Condition-Action Rules in P2P Networks, To appear in JCSS, Elsevier, 2010.

[15] D. Parker, D. Cleary. Building Richer JXTA Applications with Collaborative Spaces in a Peer-to-Peer Environment. In Proc. of the 38th Hawaii Int'l Conference on System Sciences, vol. 9, pp.301b, 2005.

[16] S. Rossi, P. Busetta: Towards Monitoring of Group Interactions and Social Roles via Overhearing. CIA 2004: 47-61.

[17] J. Schellhase, A. Frankfurth, and U. Winand. Learning scenarios for programming courses based on a CSCL and WBT support. In J. Nall and R. Robson (Eds.), Proc. of World Conference on e-Learning in Corporate, Government, Healthcare, and Higher Education, 2911-2918, 2004.

[18] M. Perez-Sanagustin, D. Hernandez-Leo, and J. Blat. Introducing Flexibility into CSCL Scripts for Blended Learning Scenarios. In Proc. of the 9th IEEE Int'l Conference on Advanced Learning Technologies, IEEE CS, p. $713,2009$.

[19] R. Shtykh, G. Zhang and Q. Jin. Peer-to-Peer Solution to Support Group Collaboration and Information Sharing. Journal Of Pervasive Computing And Communications, Vol. 1, No. 3, 2005

[20] B. Simon, Z. Mikls, W. Nejdl, M. Sintek, J. Salvachua. Smart Space for Learning: A Mediation Infrastructure for Learning Services, WWW2003, 2003.

[21] C. Steinfield, C. Jang, P. Pfaff. Supporting virtual team collaboration: The TeamSCOPE system. Proc. of the Group'99 Conference, 81-90, 1999.

[22] F. Xhafa, A. Poulovassilis. Requirements for Distributed Event-Based Awareness in P2P Groupware Systems. Proc. AINA Workshops 2010, pp 220-225.

[23] You, Y. and Pekkola, S. Meeting others -supporting situation awareness on the WWW. Decis. Support Syst. $32(1): 71-82,2001$.

[24] J. Zumbach, A. Hillers, and P. Reimann. Supporting Distributed Problem-based Learning: The Use of Feedback in Online Learning. T. Roberts (Ed.), Online Collaborative Learning: Theory and Practice, 86-103. Hershey, PA: Idea, 2003. 